\title{
Abnormal fatty acid composition in the frontopolar cortex of patients with affective disorders
}

\author{
Y Tatebayashi ${ }^{1}, \mathrm{~N}$ Nihonmatsu-Kikuchi ${ }^{1}, \mathrm{Y}$ Hayashi ${ }^{1,2}, \mathrm{X} \mathrm{Yu^{1,3 }}{ }^{\text {M Soma }}{ }^{4}$ and $\mathrm{K} \mathrm{Ikeda}{ }^{1}$
}

Bipolar and major depressive disorders are essentially relapsing and remitting disorders of affect with nearly full recovery between episodes. Although the underlying molecular mechanisms remain unclear, myelin-related abnormalities have long been suspected. Here, using novel statistical analysis, we show that subtle but significant abnormalities exist in the composition of fatty acids (FAs), including docosapentaenoic acid (22:5n-3), one of the omega-3 polyunsaturated FAs, found in the post-mortem frontopolar cortex (FPC) of subjects with bipolar or major depressive disorders, although not in those with schizophrenia. These abnormalities were all aggravated in a myelin level-dependent manner, suggesting their close relationship with myelination. Animal studies have further revealed that chronic antidepressant treatment induces robust changes in brain FA metabolism, but contributes only part of the abnormalities found in the affective disorder brains. These findings indicate that the pathophysiology of affective disorders involves an unknown type of perturbed myelination in the FPC that may serve as a novel diagnostic and therapeutic target.

Translational Psychiatry (2012) 2, e204; doi:10.1038/tp.2012.132; published online 11 December 2012

\section{Introduction}

Bipolar disorder (BD) and major depressive disorder (MDD), which are collectively known as affective disorders, are essentially relapsing and remitting disorders of affect with nearly full recovery between episodes. ${ }^{1-5}$ The frontopolar cortex (FPC, Brodmann area (BA) 10) has long been a focus of neuroimaging studies addressing these disorders. For example, longitudinal studies of MDD patients imaged both before and after various treatments have identified metabolism and flow changes in the FPC ${ }^{6-8}$ Increased metabolism in the left FPC has been associated with depression relapse during experimentally induced monoamine depletion in fullremission MDD patients, further suggesting that monoaminergic dysfunction may represent a trait abnormality in MDD and that the FPC may be involved in such pathophysiological mechanisms. ${ }^{9}$ BD patients also display blunted activation in regions adjacent to, and including, the left FPC independent of mood state. ${ }^{10}$ Other frequently reported neuroimaging abnormalities in affective disorders include white matter and subcortical gray matter hyperintensities. ${ }^{11}$

Only a few neuropathological correlates have been reported in the post-mortem FPC of subjects with affective disorders. Most of these studies demonstrated oligodendroglial abnormalities. For example, reductions in the levels of immunoreactive myelin basic protein, a marker for mature oligodendrocytes, were found in homogenates from the FPC of depressed subjects. ${ }^{12}$ Ultrastructural changes in oligodendrocytes were observed in the FPC of BD subjects. ${ }^{13}$ More recently, using a novel flow cytometric cell-counting method, Hayashi et al. ${ }^{14}$ found that the densities of oligodendrocyte- lineage (olig2-positive) cells specifically decrease in the gray matter of FPC in subjects with MDD ${ }^{14}$ and BD. ${ }^{15}$ These findings suggest that some quantitative and/or qualitative trait abnormalities in the FPC, most likely related to those of adulthood cortical myelination, may be involved in the pathophysiology of affective disorders.

Because fatty acids (FAs) are the main constituents of lipids that account for about $50-70 \%$ of the dry weight of both the human brain and myelin, respectively, ${ }^{16,17}$ abnormal FA composition may have a role in such myelin abnormalities in the FPC. To test this hypothesis, we employed a wellcharacterized set of frozen, unfixed post-mortem human brains from the Stanley Foundation Neuropathological Consortium, ${ }^{18}$ composed of FPCs and inferior temporal cortices (ITC, BA 20), from typical core cases of MDD, BD or schizophrenia (Sch) and from matched controls, and analyzed their FA composition. The ITC was included in the analysis because, in striking contrast to the FPC where adulthood intracortical myelination continues at least until the fifth decade ${ }^{19}$ the ITC may represent an exception to this process, displaying a conspicuously small degree of adulthood intracortical myelination. ${ }^{19}$

\section{Materials and methods}

All experimental protocols were approved by the Ethics Committee of the Tokyo Metropolitan Institute of Medical Science (Tokyo, Japan).

Animal studies. To investigate the effects of post-mortem interval (PMI) and frozen storage on brain FA composition,

\footnotetext{
${ }^{1}$ Affective Disorders Research Team, Schizophrenia and Affective Disorders Research Project, Tokyo Metropolitan Institute of Medical Science, Setagaya, Tokyo, Japan; ${ }^{2}$ Department of Integrative Physiology, Shiga University of Medical Science, Seta-Tsukinowa, Ohtsu, Shiga, Japan; ${ }^{3}$ Department of Neurology, Key Laboratory of Neurology of Hebei Province, Second Hospital of Hebei Medical University, Shijiazhuang, China and ${ }^{4}$ Mochida Pharmaceutical, Shinjuku, Tokyo, Japan Correspondence: Dr Y Tatebayashi, Affective Disorders Research Team, Tokyo Metropolitan Institute of Medical Science, 2-1-6 Kamikitazawa, Setagaya, Tokyo 156-8506, Japan.

E-mail: tatebayashi-ys@igakuken.or.jp

Keywords: bipolar disorder; fatty acid; frontopolar cortex; major depressive disorder; myelination; schizophrenia

Received 24 September 2012; accepted 6 October 2012
} 
8-week-old adult male wistar rats $(n=17)$ were killed by cervical dislocation. The animals were then kept at $4{ }^{\circ} \mathrm{C}$ for 0 $(n=9), 24(n=4)$ or $48 \mathrm{~h}(n=4)$ without perfusion. At each $\mathrm{PMI}$, the brain was removed from the skull and the whole cerebral cortex was dissected out as described by Hayashi et al. ${ }^{14}$ The tissues were immediately frozen and stored at $-80^{\circ} \mathrm{C}$ until measurements were carried out. The frozen brains were used within 35 days $(n=4)$, except for one group $(n=4)$ that was frozen immediately after cervical dislocation, which were stored for 550 days to investigate the effects of long-term frozen storage.

To examine the effects of myelin inclusion on FA composition, 6-week-old adult male Sprague-Dawley rats $(n=60)$ were killed under anesthesia, and the cortex was dissected without (Ex1, $n=26)$ or with (Ex2, $n=34)$ the corpus callosum, whereas the hippocampus was dissected out in its entirety $(n=60)$ as described in Supplementary Figure 2a.

To investigate the effects of medication on FA composition, 6-week-old male Sprague-Dawley rats $(n=59)$ were intraperitoneally administered saline $(n=13), 10 \mathrm{mg} / \mathrm{kg}$ amitriptyline $(n=9), 10 \mathrm{mg} / \mathrm{kg}$ fluoxetine hydrochloride $(n=4), 5 \mathrm{mg} / \mathrm{kg}$ desipramine $(n=5), 5 \mathrm{mg} / \mathrm{kg}$ setiptiline $(n=5)$ or $5 \mathrm{mg} / \mathrm{kg}$ maprotiline $(n=5)$, mood stabilizers ( $2 \mathrm{mmol} / \mathrm{kg}$ lithium chloride, $n=3 ; 200 \mathrm{mg} / \mathrm{kg}$ valproic acid, $n=3)$, psychostimulants $(5 \mathrm{mg} / \mathrm{kg}$ phencyclidine, $n=3$; $5 \mathrm{mg} / \mathrm{kg}$ methamphetamine, $n=3$ ) or antipsychotics ( $1 \mathrm{mg} /$ $\mathrm{kg}$ haloperidol, $n=3 ; 1 \mathrm{mg} / \mathrm{kg}$ risperidone, $n=3$ ) for 3 weeks. Desipramine, setiptiline and maprotiline were categorized as norepinephrine re-uptake inhibitors $(n=15)$, as they directly or indirectly inhibit norepinephrine but not serotonin reuptake, whereas amitriptyline and fluoxetine were categorized as serotonin re-uptake inhibitors $(n=13)$. Following treatment, animals were killed under anesthesia and the hippocampus and the cortex were dissected out as described in Supplementary Figure 2a. The tissues were immediately frozen, and total FA composition was determined as described below.

Post-mortem human brains. Frozen blocks of the FPC (BA 10) and ITC (BA 20) cortex were generously provided by the Stanley Foundation Neuropathology Consortium. The samples were from subjects with Sch $(n=15)$, BD $(n=15)$ and MDD without psychotic features $(n=15)$, and from normal controls $(n=15)$. With informed consent from next-of-kin, participating medical examiners collected these specimens. ${ }^{18}$ Two senior psychiatrists diagnosed these cases based on the Fourth Edition of the Diagnostic and Statistical Manual of Mental Disorders (DSM-IV) criteria and medical records, and when necessary, telephone interviews with family members. The diagnosis of unaffected controls was based on structured interviews by a senior psychiatrist with family member(s) to rule out axis I diagnosis. ${ }^{18}$ Demographic information and medical data are summarized in Table 1. The information on the medications/treatments prescribed to the patients at around the time of death is shown in Supplementary Table 1. Note, however, that these data are too incomplete and imprecise (in terms of toxicology and in other regards) to establish whether they were actually taking the medications at the time of death.
FA analysis. About $100 \mathrm{mg}$ of cortical tissue were cut from the frozen blocks, placed in $10 \times$ phosphate-buffered saline, and sonicated in a bath sonicator. All of the measurements described in this section were conducted in a blinded manner at Bio Medical Laboratories (Tokyo, Japan). Briefly, aliquots $(400 \mu \mathrm{l})$ of brain samples were taken, and the lipids were extracted by adding chloroform-methanol $\left(2: 1\left(v v^{-1}\right)\right)$. The lipid samples containing the internal standard (23:0, tricosanoid acid) were then vortexed, sonicated and centrifuged at 2000 r.p.m. for $5 \mathrm{~min}$. The organic layer was removed, and FAs were methylated with boron trifluoride and methanol. FA methyl esters were quantified by gas chromatography in a chromatograph GC-17A (Shimadzu Scientific Instruments, Kyoto, Japan) equipped with a BPX70 capillary column $(30 \mathrm{~m} \times 0.22 \mathrm{~mm}$ wide bore, $0.25 \mu \mathrm{m}$ film thickness; SGE International, Melbourne, VIC, Australia). The carrier gas was helium with a column flow rate of $0.7 \mathrm{ml} \mathrm{min}^{-1}$. The capillary gas chromatography setup and associated flow rate enabled us to detect $2 \mu \mathrm{g}$ of an individual FA in a $100 \mathrm{mg}$ brain sample. As for the accuracy of this method, the intraassay precision was $1.5-9.7 \%$ and the inter-assay precision was $3.1-17.9 \%$.

Statistical analysis. The software package JMP (version 7; SAS Institute, Cary, NC, USA) was used for all of the statistical analyses.

Multiple regression analysis was used to determine which model better explained FA concentrations: myelin inclusions in the samples or confounding factors (Supplementary Table 2). We included the following measures in the analysis: for myelin inclusion, we used FA concentrations (mol\%); and for confounding factors, we used age at death, brain $\mathrm{pH}$, brain weight, PMI (h), frozen storage (days), lifetime quantity of fluphenazine equivalent (mg), gender, suicide status, psychosis status, history of substance abuse, severity of substance abuse, severity of alcohol abuse, brain hemisphere and obesity index. Age at onset, duration of disease and smoking status were included in separate analyses because of the lack of control data or the presence of unknown status, respectively; however, none of the FA concentrations were significantly explained by any combination of confounding factors (data not shown).

Principal factor analysis was used for the extraction of most neutral predictors (myelin factor (MF)) of myelin inclusions in the cortical homogenates as described in the Results section.

We used canonical discriminant analysis to first test group differences for each uncountable confounding factor in relation to the FA concentration, and then to test each FA concentration in relation to the MF. Differences between each disease group and the control (or between each drug-treated group and the saline-treated group) were obtained by multivariate analysis of variance. $P$-values $<0.05$ were considered statistically significant.

\section{Results}

It is well known that 'free' FA content, especially that of arachidonic acid (20:4n-6), increases significantly in autopsied brains during PMls, due to high levels of lipid hydrolysis. ${ }^{20}$ This would indicate that any determinations of either 
Table 1 Demographic characteristics

\begin{tabular}{|c|c|c|c|c|c|c|}
\hline \multirow[t]{2}{*}{ Parameter } & \multirow[t]{2}{*}{ Control $(\mathrm{n}=15)$} & \multirow[t]{2}{*}{$M D D(\mathrm{n}=15)$} & \multirow[t]{2}{*}{$B D(\mathrm{n}=15)$} & \multirow[t]{2}{*}{ Schizophrenia $(\mathrm{n}=15)$} & \multicolumn{2}{|c|}{ Statistical results } \\
\hline & & & & & $\mathrm{P}$-value & Technique \\
\hline Age at death (years) & $48 \pm 11(29-68)$ & $47 \pm 9(30-65)$ & $42 \pm 12(25-61)$ & $45 \pm 13(25-62)$ & 0.540 & One-way ANOVA \\
\hline Gender & 9 Male/6 female & 9 Male/ 6 female & 9 Male/6 female & 9 Male/6 female & & \\
\hline PMI (h) & $23.7 \pm 9.9(8-42)$ & $27.5 \pm 10.7(7-47)$ & $32.5 \pm 16.1(13-62)$ & $33.7 \pm 14.6(12-61)$ & 0.147 & One-way ANOVA \\
\hline Brain $(\mathrm{pH})$ & $6.3 \pm 0.2(5.8-6.6)$ & $6.2 \pm 0.2(5.8-6.5)$ & $6.2 \pm 0.2(5.8-6.5)$ & $6.2 \pm 0.3(5.8-6.6)$ & 0.611 & One-way ANOVA \\
\hline Side of brain & 7 Right/8 left & 6 Right/9 left & 8 Right/7 left & 6 Right/9 left & 0.864 & $\chi^{2}$ test \\
\hline Brain weight (g) & $1501 \pm 164(1305-1840)$ & $1462 \pm 142(1240-1740)$ & $1441 \pm 172(1130-1690)$ & $1471 \pm 108(1270-1640)$ & 0.739 & One-way ANOVA \\
\hline Refrigeration interval (h) & $3.6 \pm 1.4(1-6)$ & $7.9 \pm 5.5(3-21)$ & $9.5 \pm 10(2-39)$ & $8.5 \pm 7.2(3-27)$ & 0.114 & One-way ANOVA \\
\hline Storage days & $2257 \pm 234(1950-2693)$ & $2353 \pm 290(2005-2850)$ & $2540 \pm 172(2143-2755)$ & $2540 \pm 233(1987-2857)$ & 0.003 & One-way ANOVA \\
\hline Age at onset (years) & - & $33.9 \pm 13.3(11-54)$ & $21.5 \pm 8.3(7-39)$ & $23.2 \pm 8.0(13-42)$ & 0.003 & One-way ANOVA ${ }^{a}$ \\
\hline Duration of disease (years) & - & $12.7 \pm 11.1(1-42)$ & $20.1 \pm 9.7(6-43)$ & $21.3 \pm 11.4(5-45)$ & 0.068 & One-way ANOVA ${ }^{a}$ \\
\hline Death by suicide & 15 No & $8 \mathrm{No} / 7 \mathrm{Yes}$ & $6 \mathrm{No} / 9$ Yes & $11 \mathrm{No} / 4 \mathrm{Yes}$ & 0.181 & ${ }^{2} \chi$ test $^{\mathrm{b}}$ \\
\hline History of psychosis & - & 15 without & 11 with $/ 4$ without & 15 with & 0.100 & Fisher's test ${ }^{c}$ \\
\hline Fluphenazine equivalent (mg) & 0 & 0 & $20827 \pm 24016(0-60000)$ & $52267 \pm 62062(0-200000)$ & 0.078 & Unpaired $t$-test ${ }^{c}$ \\
\hline \multicolumn{7}{|l|}{ Obesity index $\left(B M I, \mathrm{kgm}^{-2}\right)$} \\
\hline $15-20$ & 0 & 2 & 3 & 1 & 0.335 & $\chi^{2}$ test \\
\hline $21-25$ & 6 & 3 & 2 & 3 & & \\
\hline $26-30$ & 5 & 8 & 7 & 3 & & \\
\hline $31-35$ & 1 & 1 & 1 & 4 & & \\
\hline $36-40$ & 1 & 0 & 2 & 2 & & \\
\hline $41-45$ & 2 & 1 & 0 & 2 & & \\
\hline \multicolumn{7}{|l|}{ SUBS } \\
\hline Never & 12 & 10 & 6 & 9 & 0.377 & $\chi^{2}$ test \\
\hline Current & 1 & 4 & 6 & 4 & & \\
\hline Past & 2 & 1 & 3 & 2 & & \\
\hline \multicolumn{7}{|l|}{ SUBSSEV } \\
\hline None/little/social & 14 & 11 & 7 & 10 & 0.056 & $\chi^{2}$ test \\
\hline Moderate use (current, past) & 1 & 0 & 5 & 2 & & \\
\hline Heavy use (current, past) & 0 & 3 & 3 & 3 & & \\
\hline Unknown & 0 & 1 & 0 & 0 & & \\
\hline \multicolumn{7}{|l|}{ ETOHSEV } \\
\hline None/little/social & 11 & 9 & 4 & 7 & 0.156 & $\chi^{2}$ test \\
\hline Moderate use (current, past) & 4 & 1 & 3 & 3 & & \\
\hline Heavy use (current, past) & 0 & 4 & 5 & 3 & & \\
\hline Unknown & 0 & 1 & 3 & 2 & & \\
\hline \multicolumn{7}{|l|}{ Smoking at the time of death } \\
\hline Yes & 4 & 5 & 3 & 5 & 0.534 & $\chi^{2}$ test \\
\hline No & 3 & 5 & 7 & 7 & & \\
\hline Unknown & 8 & 5 & 5 & 3 & & \\
\hline
\end{tabular}

Abbreviations: ANOVA, analysis of variance; BD, bipolar disorder; ETOHSEV, severity of alcohol abuse; MDD, major depressive disorder; PMI, post-mortem interval; SUBS, history of substance abuse; SUBSSEV, severity of substance abuse.

Values are expressed as means \pm s.d. (minimum - maximum).

aOne-way ANOVAs were used only among MDD, BD, and schizophrenia groups. History of SUBS: never, no history of use; current, drug and/or alcohol use at the time of death; past, a history of alcohol and/or drug use but not in the 6 months before death. Group differences in smoking at the time of death were analyzed after excluding unknown subjects. ${ }^{b} \chi^{2}$ Test was used among MDD, BD and schizophrenia groups. 'Fisher's exact test and an unpaired $t$-test were used with the BD and schizophrenia groups.

lipid composition or free FAs in post-mortem brain tissues, or in the myelin isolated thereof, made using comprehensive lipidomics analyses should be undertaken with the utmost care for PMls. According to a few pioneering studies, however, 'total' brain FA composition-including that of any liberated, non-esterified FAs-tends to remain relatively stable even during long PMls. ${ }^{21,22}$ Using whole rat cerebral cortices, we confirmed that the 'total' FA composition, even for that of minor FAs $(<1.6 \mathrm{~mol} \%)$, remained unaltered during PMls (0, 24 and 48h), except for 20:4n-6 (Supplementary Figure 1a). We also found that long-term frozen storage (days) similarly had almost no effect on the 'total' FA composition, except for 20:3n-6 (Supplementary Figure 1b). Although the levels of $20: 4 n-6$ and $20: 3 n-6$, two $n-6$ polyunsaturated FAs, were significantly reduced, presumably due to the gradual conversion of these polyunsaturated FAs into eicosanoids ${ }^{23}$ during long PMIs or storage, reductions in the absolute levels remained extremely small when compared with other FA levels (Supplementary Figure 1).
In contrast, FA composition was largely influenced by the myelin inclusions. In rat brains, for example, a highly significant positive correlation was found between the concentrations of two major myelin-abundant FAs, ${ }^{17}$ oleic acid (18:1n-9) and nervonic acid (24:1n-9) (Supplementary Figures 2 and 3 ). These two FA concentrations in rat brains not only varied considerably but also increased correspondingly as myelin inclusions in the homogenates increased (Cortex $\quad($ Ex1 $)<$ Cortex $\quad($ Ex2 $)<$ Hippocampus $\quad(E x 1)=$ Hippocampus (Ex2)). This suggests that inadvertent myelin inclusions are among the most powerful factors affecting 'total' FA composition in the post-mortem rat brain.

In our own post-mortem human brain study, this proved generally true, as demonstrated by the Pearson's correlation coefficient matrix of all FA concentrations in the FPC or ITC plotted also against the available countable confounding factors (Figure 1A). While the concentrations of nine major FAs showed highly significant positive or inverse correlations with each other $(|r|>0.8)$ (multicollinearity) (Figure 1B and $\mathrm{Ca})$, none of the FA concentrations correlated to any 
A

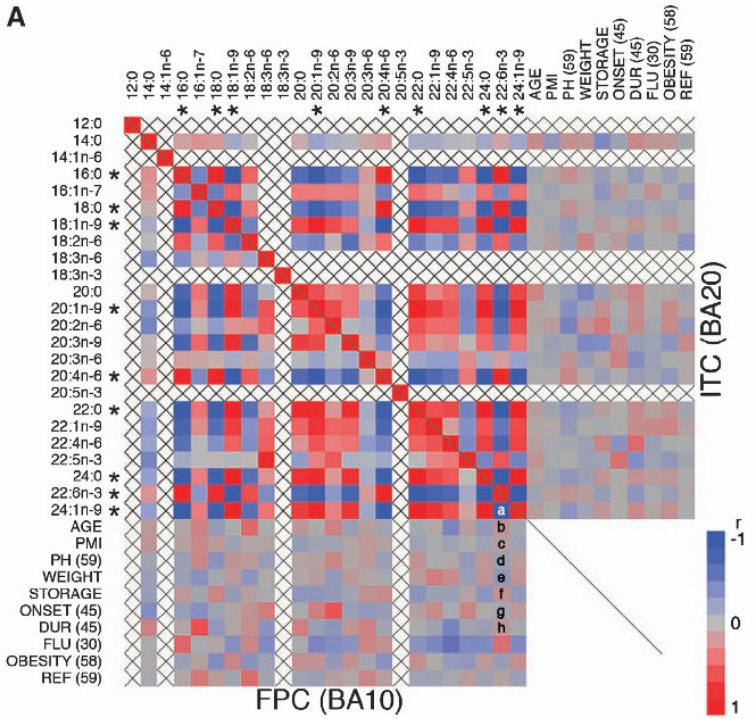

B

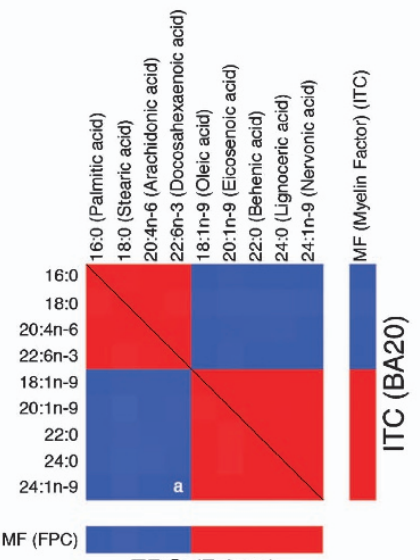

FPC (BA10)

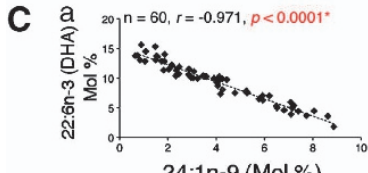

$24: 1 \mathrm{n}-9(\mathrm{Mol} \%)$

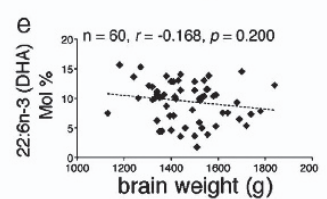

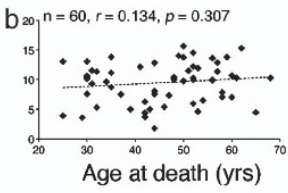

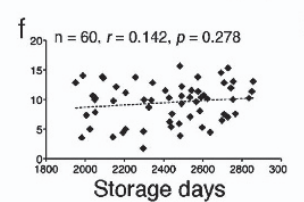

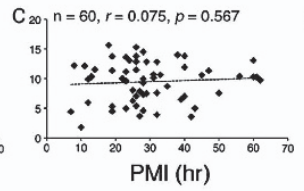
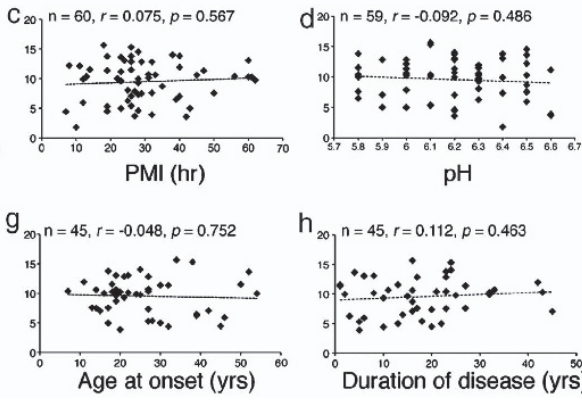

Figure 1 Effects of myelin inclusion or confounding factors on the human brain fatty acid (FA) composition. (A) Pearson's correlation coefficient matrix of 24 FAs for all available countable confounding factors of frontopolar cortex (FPC) (left, bottom) and inferior temporal cortex (ITC) (right, top) samples. Correlations are expressed by the respective color tiles. Note that none of the available countable confounding factors significantly affected FA composition, whereas the nine major FA concentrations (marked with an *) showed multicollinearity ( $|r|>0.8)$, probably due to inadvertent myelin inclusions. AGE, age at death; ONSET, age at onset; DUR, duration of disease (years); FLU, fluphenazine equivalent (mg); PH, brain pH; WEIGHT, brain weight (gram); PMI, post-mortem interval (hours); STORAGE, frozen storage (days); and OBESITY, obesity index. Letters in the matrix ( $\mathrm{A}$ and $\mathrm{B}$ ) indicate the position of graphs in panel $\mathrm{C}$ (for example, $\mathbf{a}=$ Figure $1 \mathrm{Ca}$ ). (B) Rearranged correlation coefficient matrix of the marked FAs and myelin factors (MFs) for FPC (left, bottom) and ITC (right, top) samples. (C) Correlation between 22:6n-3 (docosahexaenoic acid (DHA)) concentration (mol\%) and (a) 24:1n-9 concentration (mol\%), (b) age at death, (c) PMI, (d) pH, (e) brain weight, (f) frozen storage days, (g) age at onset and (h) duration of disease. Note that only the 24:1n-9 concentrations correlated significantly with those of DHA $(P<0.0001)$, suggesting that the concentrations of one FA (for example, DHA) can be predicted by other FA concentrations (for example, 24:1n-9) (see the results of multiple regression analyses in Supplementary Table 2).

significant degree with the available countable confounding factors (Figures $1 \mathrm{Cb}-\mathrm{h}$ ). The uncountable confounding factors also had no significant effect on the FA composition, except in terms of diagnosis, indicating that certain FA abnormalities may exist in affective disorder brains (Table 2). The sum of these nine FA concentrations accounted for about $90 \%$ of the total FA concentration (Supplementary Table 2), suggesting that an unexpectedly high proportion of FAs either increased or decreased in a corresponding manner, most likely due to the varied inclusion of myelin in the cortical homogenate (Supplementary Table 2). Furthermore, a similar multicollinearity (albeit with a somewhat different FA combination from that found in humans) was observed in the rat brains (Supplementary Figure 3), which suggests that this phenomenon may be common in animal brains containing a myelin system.

Although multicollinearity did not reduce the reliability of the model as a whole, it did affect our calculations of individual FA concentrations. Furthermore, this factor had never been seriously considered in previous post-mortem human brain studies, although some did partially note its presence. ${ }^{24}$ To untangle this multicollinearity, we conducted a principal factor analysis encompassing all 24 FA concentrations to determine the most neutral predictors of myelin proportions in the cortical homogenates. As a result, we found that Factor 1 boasted an exceptionally high eigenvalue and total variance coefficient compared with the second most powerful factor (Factor 2) (Supplementary Table 3). Moreover, it strongly correlated with the nine major FA concentrations discussed above (Figure 1B). We thus concluded that Factor 1 could most accurately predict the proportion of myelin in the cortical homogenate, and designated it as the 'MF'. We then reanalyzed all of the $\mathrm{FA}$ concentrations in relation to the calculated MFs.

Surprisingly, this novel statistical analysis revealed affective disorder-specific FA abnormalities in the FPC and ITC, 
which occurred in a myelin level-dependent manner (Figures 2 and 3 and Supplementary Tables 4 and 5). In the BD and MDD FPC, although not in the Sch or control FPC, we found that (i) two minor polyunsaturated FAs, docosapentaenoic $(22: 5 n-3)$ and $\gamma$-linolenic $(18: 3 n-6)$ acids, abnormally

Table 2 Effects of uncountable confounding factors on the human brain FA composition

\begin{tabular}{|c|c|c|c|c|c|c|}
\hline \multirow[t]{2}{*}{ Parameter } & \multicolumn{3}{|c|}{$F P C(B A 10)$} & \multicolumn{3}{|c|}{ ITC (BA 20) } \\
\hline & $\begin{array}{c}\text { Wilks' } \\
\lambda\end{array}$ & $F$ & P-value & $\begin{array}{c}\text { Wilks' } \\
\lambda\end{array}$ & $F$ & $\begin{array}{c}\mathrm{P} \text { - } \\
\text { value }\end{array}$ \\
\hline Gender (60) & 0.678 & 0.692 & 0.826 & 0.692 & 0.649 & 0.865 \\
\hline Side of Brain (60) & 0.546 & 1.212 & 0.296 & 0.591 & 1.011 & 0.479 \\
\hline $\begin{array}{l}\text { Death by Suicide } \\
(60)\end{array}$ & 0.572 & 1.091 & 0.399 & 0.570 & 1.101 & 0.391 \\
\hline SUBS (60) & 0.531 & 0.528 & 0.212 & 0.502 & 0.582 & 0.975 \\
\hline SUBSSEV (59) & 0.521 & 0.530 & 0.989 & 0.492 & 0.586 & 0.973 \\
\hline ETOHSEV (54) & 0.331 & 0.861 & 0.701 & 0.339 & 0.838 & 0.734 \\
\hline Psychosis (60) & 0.641 & 0.816 & 0.695 & 0.709 & 0.600 & 0.903 \\
\hline Diagnosis (60) & 0.023 & 3.489 & $<0.0001^{*}$ & 0.094 & 1.662 & $0.01^{\star}$ \\
\hline Sch vs Cont (30) & 0.263 & 0.583 & 0.831 & 0.419 & 0.289 & 0.989 \\
\hline AD vs Cont (45) & 0.061 & 12.808 & $<0.0001^{*}$ & 0.204 & 3.253 & $0.005^{\star}$ \\
\hline AD vs Sch (45) & 0.054 & 14.667 & $<0.0001^{*}$ & 0.176 & 3.894 & $0.002^{\star}$ \\
\hline BD vs MDD (30) & 0.177 & 0.969 & 0.579 & 0.307 & 0.470 & 0.904 \\
\hline
\end{tabular}

Abbreviations: $A D$, affective disorders (including $B D$ and $M D D$ ); $B D$, bipolar disorder; Cont, normal control; ETOHSEV, severity of alcohol abuse; FA, fatty acid; FPC, frontopolar cortex; ITC, inferior temporal cortex; MDA, multiple discriminant analysis; MDD, major depressive disorder; Sch, schizophrenia; SUBS, history of substance abuse; SUBSSEV, severity of substance abuse. MDA was used for the analyses. $P$-values $\leq 0.05$ are shown in bold. Numbers within parentheses indicate numbers of samples analyzed. accumulated (Figures 2a and b), and (ii) the level of 18:1 $n-9$, the ratio of $18: 1 n-9 / 18: 0$ (stearic acid) (presumably representing stearoyl-coenzyme $A$ desaturase activity) and the ratio of 20:0 (arachidic acid)/18:0 (presumably representing elongase activity) significantly decreased (Figures $2 c-e$ ). Furthermore, all of these abnormalities were more extreme in those samples with higher MFs (that is, those with more myelin).

In the ITC, in contrast, the levels of $22: 5 n-3$ highly significantly decreased in the affective disorder subjects (Figure $3 \mathrm{a}$ and Supplementary Table 4). Similar changes were found in the hippocampus (Figure $3 b$ ) and in the cortex (data not shown) of rats chronically treated with antidepressants, suggesting that medication and/or some compensatory mechanisms may be involved in the FA abnormalities found in the affective disorder ITC. Chronic antidepressant treatment also induced the upregulation of ratios of $20: 1 n-9$ (gondoic acid)/18:1n-9 in the rat brains, an abnormality also found in the affective disorder FPC (Figures $3 c$ and $d$ and Supplementary Table 5). However, none of the top five FA metabolic abnormalities found in the affective disorder FPC (Figure 2a-e) was recapitulated in the rat brains (Supplementary Tables 6 and 7 ), suggesting that medications may have only limited effects on the major FA metabolic abnormalities found in the affective disorder FPC.

Post hoc analysis further revealed that none of the confounding factors (for example, left hemisphere vs right hemisphere, male vs female, and so on) influenced the abnormal FA metabolic abnormalities found in the affective disorder brains (data not shown).
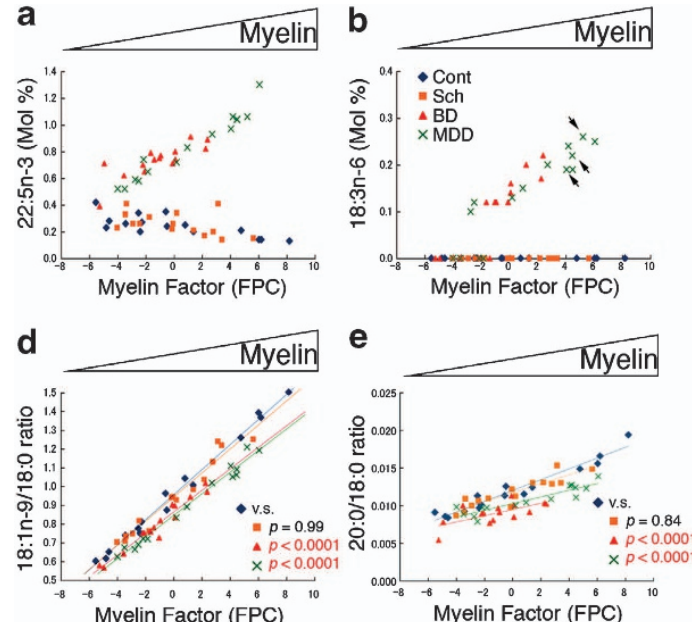

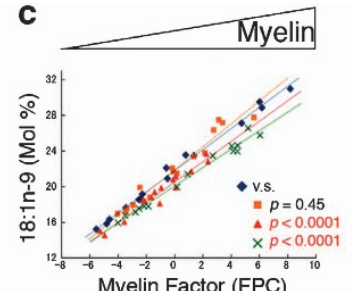
Myelin Factor (FPC)

$c^{\prime}$

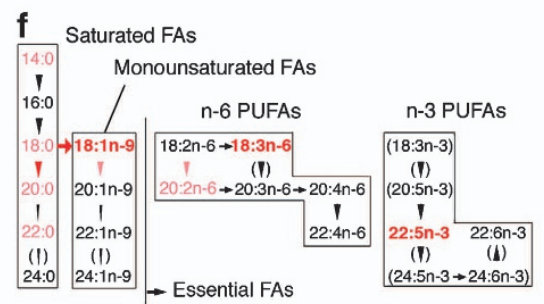

Figure 2 Top five fatty acid (FA) metabolic abnormalities in the affective disorder frontopolar cortex (FPC). (a) 22:5n-3. As the myelin factors (MFs) (myelin proportions in the cortical homogenates) increased, 22:5n-3 accumulated in the affective disorder (bipolar disorder (BD), major depressive disorder (MDD)), but not in the schizophrenia (Sch) FPC samples. (b) 18:3n-6. Similar accumulation of 18:3n-6 was observed only in the affective disorder FPC samples. Arrows indicate data from subjects with MDD who had not been prescribed antidepressant medications at around the time of death (see Supplementary Table 1), suggesting that such medications may not affect these findings. (c) 18:1n-9. Myelin-abundant FA, 18:1n-9, was significantly reduced only in the affective disorder FPC samples, particularly those having more myelin. (c') Conventional comparison of $18: 1 n-9$ concentrations. Note that, without MFs, we were deprived of information regarding the relationship between myelin and FA concentration. Furthermore, a statistically significant difference was only found between Sch and BD ( $P=0.03)$. (d) The 18:1n-9/18:0 ratios. (e). The 20:0/18:0 ratios. These two ratios were decreased in the mood disorder FPC samples, especially those having more myelin. (f) Summary of the FA metabolic abnormalities in the affective disorder FPC samples. Top five abnormalities found in this study are shown in red. Other significant, but less specific FAs, as well as their corresponding metabolic abnormalities, were also found in the affective disorder FPC samples (colored pink, see Supplementary Tables 4 and 5). Arrows indicate desaturase activities, downward arrowheads elongase activities, and an upward arrowhead $\beta$-oxidation. Several FA concentrations or their metabolic activities were undetectable in this study, and are therefore parenthesized. PUFAs, polyunsaturated FAs. 

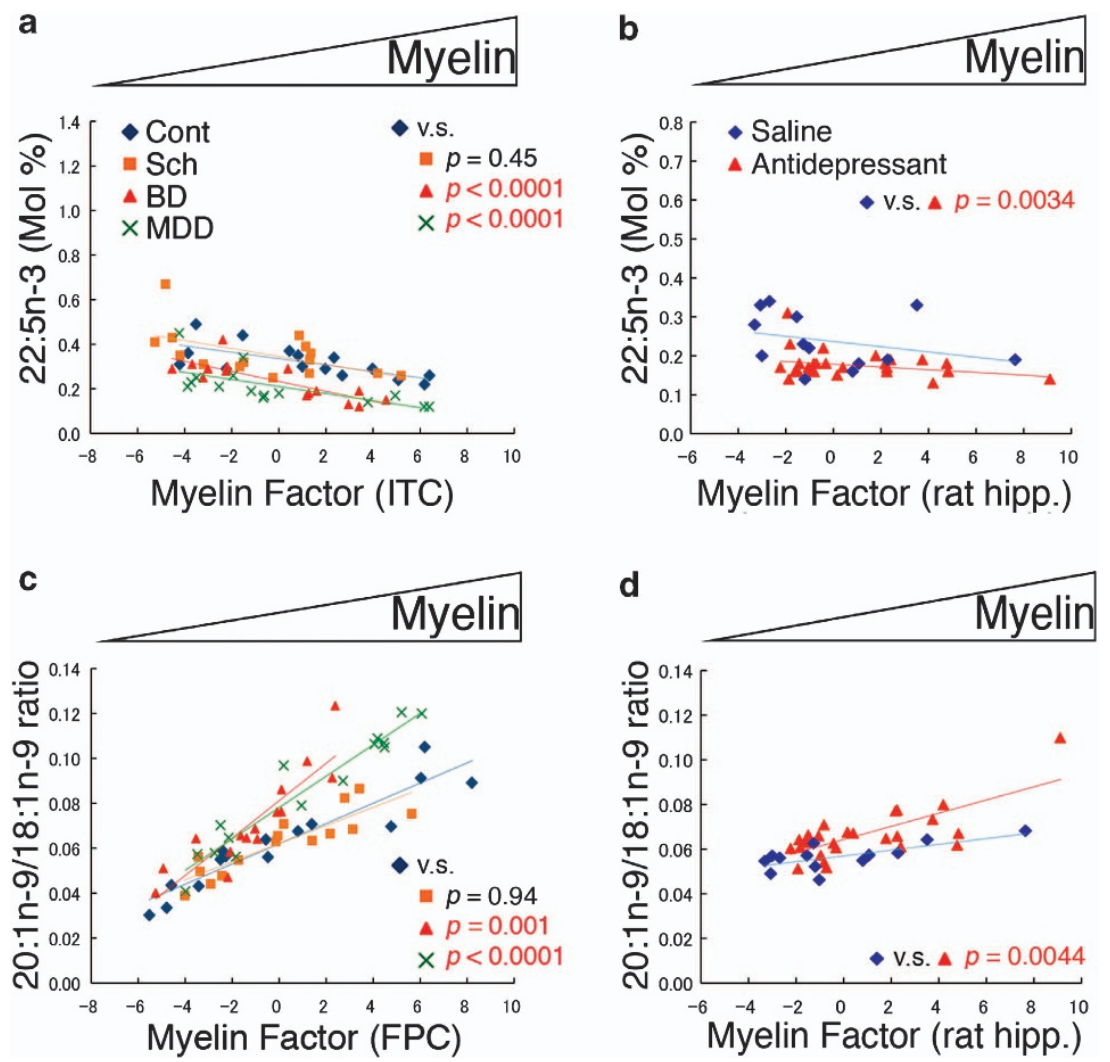

Figure 3 The similar fatty acid (FA) metabolic abnormalities in affective disorder brains and rat hippocampi chronically treated with antidepressants. (a) 22:5n-3 in the inferior temporal cortex (ITC). In contrast to the frontopolar cortex (FPC) (Figure 2a), the concentration of 22:5n-3 decreased significantly in the affective disorder ITC. (b) 22:5n-3 in the rat hippocampus chronically treated with or without antidepressants. Chronic antidepressant treatment significantly reduced the concentrations of $22: 5 n-3$ in the rat hippocampus. (c, d) 20:1n-9/18:1n-9 ratios in the FPC and rat hippocampus. As the myelin fibers (MFs) (myelin proportions in the cortical homogenates) increased, these ratios increased and became abnormally upregulated in the affective disorder FPC (c), as well as in the rat hippocampus chronically treated with antidepressants (d).

\section{Discussion}

In this study, we initially found that inadvertent myelin inclusion, but not the confounding factors, was the single most powerful factor affecting 'total' FA composition in the post-mortem human cortical homogenates, resulting in highly significant multicollinearity among the major FA concentrations. To untangle this multicollinearity, we then statistically generated an 'MF' representing the most unbiased and redundant predictor of each myelin proportion of the cortical homogenate, and re-analyzed all of the FA concentrations in relation to these factors. We found that highly significant FA metabolic abnormalities existed in a myelin level-dependent manner in the affective disorder FPC (Figure 2f).

We focused on the results from the FPC, but not on those from the ITC in this study, as the main FA abnormality found in the affective disorder ITC could be reproduced in the rat brains chronically treated with antidepressants (22:5n-3; Figures 3a and $b$ ), while the top five FA abnormalities found in the affective disorder FPC (Figure 2f) could not. These findings further illuminated the important roles played by the FPC as part of the neurocircuitry involved in the pathophysiology of affective disorders. The human FPC is unique in several respects. First, it is the largest single architectonic region of the human prefrontal cortex. ${ }^{25}$ Second, it is disproportionately larger in the human brain than in any other animals, including the apes. ${ }^{26}$ Third, it is the only prefrontal region that is almost exclusively connected to the more posterior supramodal areas, such as the dorsolateral prefrontal (BA 9, 46) and orbitofrontal cortices (BA 11, 12, 13 and 47). ${ }^{27,28}$ In addition, its projections are broadly glutamatergic and GABAergic, as well as reciprocal. ${ }^{27,29}$ Fourth, it is probably one of the last brain regions to achieve myelination. ${ }^{18,30}$

The amounts of myelin, the single most powerful factor affecting total FA composition in the cortical homogenates, can be varied not only by sampling but also by the presence of intracortical myelination. ${ }^{18,30}$ Thus, if disease pathogenesis can affect myelination in simply quantitative terms (for example, reduced myelination without any FA metabolic abnormalities), changes in the FA composition in a disease group would be indistinguishable from those induced by sampling in a control group. This concept may well explain the findings in the Sch cases. Although myelin-related abnormalities have been implicated in Sch, ${ }^{31}$ they might be quantitative, at least in the FPC and ITC. However, if disease pathogenesis influences myelination in qualitative terms, then changes in the FA composition should be detectable in a myelin level-dependent manner and this may well explain the findings in the affective disorder brains. 
So what types of qualitative abnormalities can occur in FA metabolism in affective disorder FPC? With regard to the biosynthesis of FAs or lipids, the endoplasmic reticulum may be a common organelle related to the top five FA metabolic abnormalities found in the affective disorder FPC. For example, $18: 3 n-6,22: 5 n-3$ and $18: 1 n-9$ can serve as components of glycerophospholipids $^{17,32}$ synthesized on the cytoplasmic side of the endoplasmic reticulum. Stearoyl-coenzyme A desaturases and elongases are also known to be the endoplasmic reticulum enzymes ${ }^{33,34}$ whose expression is abundant in glial cells but almost negligible in neurons in the adult rodent brain. ${ }^{35}$ A possible explanation linking our findings and recent advances might be that a type of endoplasmic reticulum stress exists in the oligodendrocyte lineage cells in the affective disorder FPC due to the prolonged dysregulation of growth factor systems, such as those of fibroblast growth factors. ${ }^{36,37}$

Clinically, this finding is highly intriguing. First, our findings clearly support, in biological terms, the current series of diagnostic classifications (DSM-IV) originally drawn from the Kraepelin's dichotomy. ${ }^{1}$ Second, affective disorders (including MDD and BD) may be a type of myelin and/or myelination disorders that occurs in specific parts of the brain, such as the FPC, thus shedding light on their relationship with the relapsingremitting type of multiple sclerosis, a major neurological myelin disorder characterized also by a high prevalence of affective symptoms. ${ }^{38}$ Third, the implicated myelin abnormalities in the Sch brains, ${ }^{31}$ if they indeed exist, may be quantitative but not qualitative in nature. Sch, which is characterized by relentlessly deteriorating mental functions and psychosis, might actually be a disease involving failure in neurons and/or neuronal connections, as we recently found, using a novel flow cytometric cellcounting method, that the densities of small NeuN-positive neurons (most likely representing small interneurons) in the gray matter of Sch FPC and ITC were decreased without any concomitant loss of total NeuN-positive neuron densities. ${ }^{15}$

The exact functions of the FPC remain enigmatic, putatively ranging from planning to self-initiated behavior, social cognition, task switching and memory. ${ }^{27,28,39}$ Given that the FPC is thought to be the most evolutionarily recent expansion of the primate prefrontal cortex, its function may uniquely reflect human adaptations in the context of selecting and updating models of reward contingency in dynamic environments. As adulthood intracortical myelination, which is influenced by both genetic and environmental factors, is an essential process for the establishment of efficient neuronal signaling networks, ${ }^{40}$ any underlying abnormalities in the FPC may have an important role in the pathophysiology of affective disorders via biological pathways that have yet to be elucidated. A better understanding of these phenomena will provide important insights to facilitate the more effective diagnosis, treatment and prevention of affective disorders.

\section{Conflict of interest}

The authors declare no conflict of interest.

Acknowledgements. Post-mortem brains were donated by the Stanley Foundation Brain Collection, courtesy of Drs Michael B Knable, E Fuller Torrey, Maree J Webster and Robert H Yolken. We thank Drs Yoshihide Kanaoka, Tomohito Hamazaki, Kikuko Hotta, Makoto Murakami and Tadafumi Kato for critical discussion of the manuscript. We thank Mr Seiichi Haga and Mses Takiko Shinozaki and Taeko Itou for their technical assistance. YT was supported by a Grant-in-Aid for Scientific Research from the Japan Society for the Promotion of Science (Tokyo, Japan).

1. Kraepelin E. Psychiatrie: ein lehrbuch fur studierende und arzte, 6. Aufl. Barth: Leipzig, 1899.

2. Goodwin FK, Jamison KR. The natural course of manic-depressive illness. In: Post RM, Ballenger JC (eds.) Neurobiology of Mood Disorders: Frontiers of Clinical Neuroscience Vol 1 (Williams \& Wilkins: Baltimore, MD, 1984. pp 20-37.

3. Judd LL, Akiskal HS, Maser JD, Zeller PJ, Endicott J, Coryell W et al. A prospective 12-year study of subsyndromal and syndromal depressive symptoms in unipolar major depressive disorders. Arch Gen Psychiatry 1998; 55: 694-700.

4. Judd LL, Akiskal HS, Schettler PJ, Endicott J, Maser JD, Solomon DA et al. The long-term natural history of the weekly symptomatic status of bipolar I disorder. Arch Gen Psychiatry 2002; 59: 530-537.

5. Judd LL, Akiskal HS, Schettler PJ, Coryell W, Endicott J, Maser JD et al. A prospective investigation of the natural history of the long-term weekly symptomatic status of bipolar II disorder. Arch Gen Psychiatry 2003; 60: 261-269.

6. Drevets WC, Videen TO, Price JL, Preskorn SH, Carmichael ST, Raichie ME. A functional anatomical study of unipolar depression. J Neurosci 1992; 12: 3628-3641.

7. Mayberg HS, Lozano AM, Voon V, McNeely HE, Seminowicz D, Hamani C et al. Deep brain stimulation for treatment-resistant depression. Neuron 2005; 45: 651-660.

8. Goldapple K, Segal Z, Garson C, Lau M, Bieling P, Kennedy S et al. Modulation of corticallimbic pathways in major depression: treatment-specific effects of cognitive behavior therapy. Arch Gen Psychiatry 2004; 61: 34-41.

9. Hasler G, Fromm S, Carlson PJ, Luchenbaugh DA, Waldeck T, Geraci M et al. Neural response to catecholamine depletion in unmedicated subjects with major depressive disorder in remission and healthy subjects. Arch Gen Psychiatry 2008; 65: 521-531.

10. Blumberg HP, Leung HC, Skudlarski P, Lacadie CM, Fredericks CA, Harris BC et al. A functional magnetic resonance imaging study of bipolar disorder: state- and trait-related dysfunction in ventral prefrontal cortices. Arch Gen Psychiatry 2003; 60: 601-609.

11. Kempton MJ, Salvador Z, Munafò MR, Geddes JR, Simmons A, Frangou S et al. Structural neuroimaging studies in major depressive disorder. Meta-analysis and comparison with bipolar disorder. Arch Gen Psychiatry 2011; 68: 675-690.

12. Honer WG, Falkai P, Chen C, Arango V, Mann JJ, Dwork AJ. Synaptic and plasticityassociated proteins in anterior frontal cortex in severe mental illness. Neuroscience 1999; 91: $1247-1255$.

13. Uranova N, Orlovskaya D, Vikhreva O, Zimina I, Kolomeets N, Vostrikov V et al. Electron microscopy of oligodendroglia in severe mental illness. Brain Res Bull 2001; 55: 597-610.

14. Hayashi Y, Nihonmatsu-Kikuchi N, Yu X-J, Ishimoto K, Hisanaga S-I, Tatebayashi Y. A novel, rapid, quantitative cell-counting method reveals oligodendroglial reduction in the frontopolar cortex in major depressive disorder. Mol. Psychiatry 2011; 16: 1156-1158.

15. Hayashi Y, Nihonmatsu-Kikuchi N, Hisanaga S-I, Yu X-J, Tatebayashi Y. Neuropathological similarities and differences between schizophrenia and bipolar disorder: a flow cytometric postmortem brain study. PLoS One 2012; 7: e33019.

16. O'Brien JS, Sampson EL. Lipid composition of the normal human brain: gray matter, white matter, and myelin. J Lipid Res 1965; 6: 537-544.

17. O'Brien JS, Sampson EL. Fatty acid and fatty aldehyde composition of the major brain lipids in normal human gray matter, white matter, and myelin. J Lipid Res 1965; 6: 545-551.

18. Braak H, Braak E. Development of Alzheimer-related neurofibrillary changes in the neocortex inversely recapitulates cortical myelogenesis. Acta Neuropathol 1996; 92: 197-201.

19. Torrey EF, Webster M, Knable M, Johnston N, Yolken RH. The Stanley Foundation Brain Collection and Neuropathology Consortium. Schizophr Res 2000; 44: 151-155.

20. Marion J, Wolfe LS. Origin of the arachidonic acid released post-mortem in rat forebrain Biochim Biophys Acta 1979; 574: 25-32.

21. Carver JD, Benford VJ, Han B, Cantor AB. The relationship between age and the fatty acid composition of cerebral cortex and erythrocytes in human subjects. Brain Res Bull 2001; 56: 79-85.

22. Fraser $\mathrm{T}$, Tayler $\mathrm{H}$, Love S. Low-temperature improved-throughput method for analysis of brain fatty acids and assessment of their post-mortem stability. J Neurosci Methods 2008; 169: 135-140.

23. Funk DC. Prostaglandins and leukotrienes: advances in eicosanoid biology. Science 2001; 294: $1871-1875$.

24. McNamara RK, Hahn CG, Jandacek R, Rider T, Tso P, Stanford KE et al. Selective deficits in the omega-3 fatty acid docosahexaenoic acid in the postmortem orbitofrontal cortex of patients with major depressive disorder. Biol Psychiatry 2007; 62: 17-24.

25. Ongür D, Ferry AT, Price JL. Architectonic subdivision of the human orbital and medial prefrontal cortex. J Comp Neurol 2003; 460: 425-449.

26. Semendeferi K, Armstrong E, Schleicher A, Zilles K, Van Hoesen GW. Prefrontal cortex in humans and apes: a comparative study of area 10. Am J Phys Anthropol 2001; 114: 224-241.

27. Koechlin E, Hyafil A. Anterior prefrontal function and the limits of human decision-making. Science 2007; 318: 594-598. 
28. Ramnani N, Owen AM. Anterior prefrontal cortex: insights into function from anatomy and neuroimaging. Nat Rev Neurosci 2004; 5: 184-194.

29. Passingham RE, Stephan KE, Kötter R. The anatomical basis of functional localization in the cortex. Nat Rev Neurosci 2002; 3: 606-616.

30. Kaes T. Die Grosshirnrinde des Menschen in ihren Massen und in ihrem Fasergehalt. Fischer: Jena, 1907.

31. Davis KL, Stewart DG, Friedman JI, Buchsbaum M, Harvey PD, Hof PR et al. White matter changes in schizophrenia. Evidence for myelin-related dysfunction. Arch Gen Psychiatry 2003; 60: 443-456

32. Martínez M, Mougan I. Fatty acid composition of human brain phospholipids during normal development. J Neurochem 1998; 71: 2528-2533.

33. Flowers MT, Ntambi JM. Role of stearoyl-coenzyme A desaturase in regulating lipid metabolism. Curr Opin Lipidol 2008; 19: 248-256.

34. Jakobsson A, Westerberg R, Jacobsson A. Fatty acid elongases in mammals: their regulation and roles in metabolism. Prog Lipid Res 2006; 45: 237-249.

35. Cahoy JD, Emery B, Kaushal A, Foo LC, Zamanian JL, Christopherson KS et al. A transcriptome database for astrocytes, neurons, and oligodendrocytes: a new resource for understanding brain development and function. J Neurosci 2008; 28: 264-278.

36. Kaga Y, Shoemaker WJ, Furusho M, Bryant M, Rosenbluth J, Pfeiffer SE et al. Mice with conditional inactivation of fibroblast growth factor receptor-2 signaling in oligodendrocytes have normal myelin but display dramatic hyperactivity when combined with Cnp1 inactivation. J Neurosci 2006; 26: 12339-12350.

37. Elsayed M, Banasr M, Duric V, Fournier NM, Licznerski P, Duman RS. Antidepressan effects of fibroblast growth factor-2 in behavioral and cellular models of depression. Biol Psychiatry 2012; 72: 258-265.

38. Passamonti L, Cerasa A, Liguori M, Gioia MC, Valentino P, Nisticò R et al. Neurobiological mechanisms underlying emotional processing in relapsing-remitting multiple sclerosis. Brain 2009; 132: 3380-3391.

39. Daw ND, O'Doherty JP, Dayan P, Seymour B, Dolan RJ. Cortical substrates for exploratory decisions in humans. Nature 2006; 441: 876-879.

40. Nave KA, Trapp BD. Axon-glial signaling and the glial support of axon function. Annu Rev Neurosci 2008; 31: 535-561.

(c)

Translational Psychiatry is an open-access journal published by Nature Publishing Group. This work is licensed under the Creative Commons Attribution-NonCommercialShare Alike 3.0 Unported License. To view a copy of this license, visit http://creativecommons.org/licenses/by-nc-sa/3.0/

Supplementary Information accompanies the paper on the Translational Psychiatry website (http://www.nature.com/tp) 